\title{
Relative entropy for compact Riemann surfaces
}

\author{
J. Gaite \\ Instituto de Matemáticas y Física Fundamental, CSIC, Serrano 123, 28006 Madrid, Spain \\ and Laboratorio de Astrofísica Espacial y Física Fundamental, Apartado 50727, 28080 Madrid, Spain
}

(Received 28 May 1999; published 13 March 2000)

\begin{abstract}
The relative entropy of the massive free bosonic field theory is studied on various compact Riemann surfaces as a universal quantity with physical significance, in particular, for gravitational phenomena. The exact expression for the sphere is obtained, as well as its asymptotic series for large mass and its Taylor series for small mass. One can also derive exact expressions for the torus but not for higher genus. However, the asymptotic behavior for large mass can always be established—up to a constant—with heat-kernel methods. It consists of an asymptotic series determined only by the curvature-and, hence, is common for homogeneous surfaces of genus higher than one-and exponentially vanishing corrections whose form is determined by the concrete topology. The coefficient of the logarithmic term in this series gives the conformal anomaly.

PACS number(s): 04.62.+v, 11.10.Gh, 11.10.Kk
\end{abstract}

\section{INTRODUCTION}

The entropy of a statistical model relative to its critical point has been shown to be an interesting quantity in field theory, especially in regard to the renormalization group [1]. On the one hand, it exhibits better behavior than the free energy when the ultraviolet cutoff is sent to infinity and, on the other hand, it is monotonic with the coupling constants, unlike the free energy. This second property makes it suitable to embody the irreversible nature of the renomalization group, which can in particular be substantiated in a finite geometry as monotonicity with respect to its characteristic scale [2]. The computation of the relative entropy for various models on a cylinder clearly shows its monotonicity [2]. The cylinder is appropriate to illustrate finite size effects but it may not be the finite geometry of choice in the context of renormalization-group irreversibility. There is a celebrated result on renormalization-group irreversibility in twodimensional (2D) field theories, Zamolodchikov $C$ theorem. The monotonicity theorem for the relative entropy on the cylinder, once it is conveniently formulated, resembles Zamolodchikov $C$ theorem [2]. However, this resemblance can hardly lead to a direct relationship, since the proof of Zamolodchikov $C$ theorem demands rotation as well as translation symmetry. In other words, that proof demands to consider a maximally symmetric space, namely, the sphere, the plane, or the hyperbolic plane. Both the sphere and the hyperbolic plane possess an infrared scale, the curvature radius, but only the sphere is finite and therefore the computation of the relative entropy on the sphere is of particular value.

From a different point of view, the calculation of the spectrum of the Laplacian operator on general compact Riemann surfaces has first held the interest of mathematicians $[3,4]$ and second of physicists $[5,6]$ for some time. The partition function of the bosonic massive field theory on a compact Riemann surface is a global object which can be constructed from the knowledge of the spectrum of the Laplacian operator. Since this spectrum is highly dependent on the topologic and geometric properties of the Riemann surface, these properties are reflected by the partition func- tion. However, this function, or say the free energy, is ultraviolet (UV) divergent and hence ill defined. Fortunately, the relative entropy of the $2 \mathrm{D}$ bosonic massive field theory is UV finite [2] and so it is likely to have a role in the geometrical characterization of a Riemann surface. This characterization consists of local parameters (the curvature) and global parameters, specifying the boundary conditions and being topologically significant. The study of homogeneous surfaces will provide insight into the dependence of the relative entropy on both types of parameters.

Generically, the relative entropy is not related to the quantum field theory entropy, but there is a direct relation on the torus (or cylinder) geometry. The relative entropy is a geometric entropy, of the sort already considered in connection with the entropy of black holes [7]. Indeed, the geometry relevant to this case is that of the cone, which is noncompact and is actually related to the cylinder geometry, and hence to the usual quantum field theory entropy, as analyzed in Ref. [2]. One can expect that the relative entropy for homogeneous spaces will be applicable in a cosmological context, once suitably generalized. Thus the results of this paper must have some bearing on entropic considerations in de Sitter and anti-de Sitter space-time. An attempt at introducing the maximum entropy principle in quantum cosmology has been made in Ref. [8]. On the other hand, the application of scaling and renormalization group concepts in gravitation [9] and cosmology [10] is gaining momentum. Therefore, it seems interesting to study properties of the entropy relative to the scales defining some curved space. Furthermore, the role of the relative entropy as a monotonic function with the renormalization group may have some relevance in modern theories of quantum gravity, as recent work seems to indicate [11].

Therefore, our main concern here will be the computation of the universal relative entropy of the 2D bosonic massive field theory on homogeneous and compact Riemann surfaces, for its own sake and with a view to its application in connection with Zamolodchikov $C$ theorem. For the plane and cylinder, the relative entropy has been computed in Ref. [2]. Since we are now concerned with compact Riemann surfaces, we shall first focus on the simplest case, namely, 
the sphere. In general, the topological classification of compact Riemann surfaces is given by their genus, that is, the number of handles in a three-dimensional embedding. The genus of the sphere is zero, of course. Compact Riemann surfaces of higher genus, with zero or negative constant curvature, will also be considered here, even though they are not globally isotropic, since they are derived from the plane or the hyperbolic space by imposing boundary conditions on a finite domain, which break rotation invariance. The case of zero curvature is the torus and is actually related to the cylinder, treated in Ref. [2]. Compact Riemann surfaces of genus $g>1$ are always related to the hyperbolic plane. The spectrum of the Laplacian on them is too complicated to allow derivation of closed expressions for the relative entropy. Thus, the case $g>1$ will be discussed summarily and only some general properties of the free energy and the relative entropy will be extracted. The method used to obtain these properties, namely, the heat-kernel method, is however of general interest and we shall dedicate considerable attention to it.

A great amount of literature has been devoted to the computation of vacuum energy densities on various manifolds, mostly in regard to field theory in curved space-time and to the Casimir effect. That energy is divergent, of course, and needs regularization. The usual technique is the zeta-function regularization [12-14], as introduced earlier in the mathematical literature [4]. It is related to the heat-kernel representation that will be utilized here by a Mellin transform. We will see how this relationship materializes for $g>1$ Riemann surfaces in the last section. ${ }^{1}$ However, since the relative entropy is a universal quantity we do not need to bother with prescribing any regularization method and we shall only do it to make connections, for instance, with the conformal anomaly or with partition functions in string theory.

\section{THE RELATIVE ENTROPY OF THE SPHERE}

The free energy $W$, namely, minus the logarithm of the partition function, for the cutoff bosonic massive field theory can be expressed as $[1,2]$

$$
W[m, \Lambda] \equiv-\ln Z[m, \Lambda]=\frac{1}{2} \sum_{\vec{p}} \ln \frac{\vec{p}^{2}+m^{2}}{\Lambda^{2}} .
$$

The set of momenta to be summed depends on the type of geometry and is such that $\vec{p}^{2}<\Lambda^{2}$. If we try to remove the cutoff we see that $W$ is UV divergent, and hence it is nonuniversal. The eigenvalues of the Laplacian on the sphere are well known, namely, $\vec{p}^{2}=l(l+1) / R^{2}$, where $R$ is the sphere radius. Thus, the sum over momenta is a sum over $l$ and we write

$$
W[r, \Lambda]=\frac{1}{2} \sum_{l=1}^{l_{\max }}(2 l+1) \ln \frac{l(l+1)+r}{(\Lambda R)^{2}},
$$

\footnotetext{
${ }^{1}$ For a comprehensive review of all these techniques, see Ref. [15].
}

where we have introduced the dimensionless coupling $r$ $=(m R)^{2}$ and the UV cutoff is related to the maximum value of $l, \Lambda \approx l_{\max } / R$. We have also removed the zero mode $l$ $=0$ from the sum, which is not allowed for $r=0$, and we have taken the degeneracy into account by the factor $2 l+1$.

From the previous expression of $W$ one can obtain the relative entropy as a universal quantity, that is, as a convergent series in the limit $l_{\max } \rightarrow \infty$. However, the sum of that series is very hard to carry out, so we choose another way. One can lower the degree of divergence of $W$ by taking derivatives with respect to $r$. In fact,

$$
\frac{d W}{d r}=\frac{1}{2} \sum_{l=1}^{l_{\max }} \frac{2 l+1}{l(l+1)+r},
$$

which is still logarithmically divergent. We can remove this divergence just by substracting its value at $r=0$ and write, in the limit $l_{\max } \rightarrow \infty$,

$$
\frac{d W}{d r}-\left.\frac{d W}{d r}\right|_{r=0}=-\frac{r}{2} \sum_{l=1}^{\infty} \frac{2 l+1}{[l(l+1)+r] l(l+1)} .
$$

Hence, we define the function $U(r):=(d W / d r)$ $-(d W / d r)_{r=0}$, which turns out to be computable in terms of the digamma function $\psi(x)$. The full expression is rather long; it is given in the Appendix.

The function $U(r)$ has interest on its own, since it is the substracted energy, but we are more interested in the relative entropy. This can be expressed in terms of $U(r)$ as

$$
S(r)=W(r)-W(0)-r \frac{d W(r)}{d r}=\int_{0}^{r} U(s) d s-r U(r) .
$$

Unfortunately, this integral cannot be done in closed form. However, it is possible to establish the behavior of $S(r)$ for small or large $r$. For large $r$ the correlation length is much smaller than the radius of the sphere and the result in the plane, $S(r)=r /(8 \pi)$, should be relevant. As $R \rightarrow \infty$ the sum over $l$ can be substituted by an integral,

$$
\sum_{l=1}^{\infty}(2 l+1) \rightarrow A \int_{0}^{\infty} \frac{d^{2} p}{(2 \pi)^{2}},
$$

where the area of the sphere is $A=4 \pi R^{2}$. We see that to compare with the value in the plane we must multiply this value by $4 \pi$. To extract the dominant large $r$ behavior of $U(r)$, we use $\psi(x) \approx \ln x$. A lengthy but straightforward calculation shows that $U(r) \approx-\ln r / 2$. Hence, using first an integration by parts,

$$
S(r)=-\int_{0}^{r} s U^{\prime}(s) d s \approx \frac{r}{2},
$$

as expected.

The full asymptotic expansion of $S(r)$ near infinity results from that of $U(r)$, which in turn can be worked out with the help of the known asymptotic expansion of $\psi(x)$. However, 

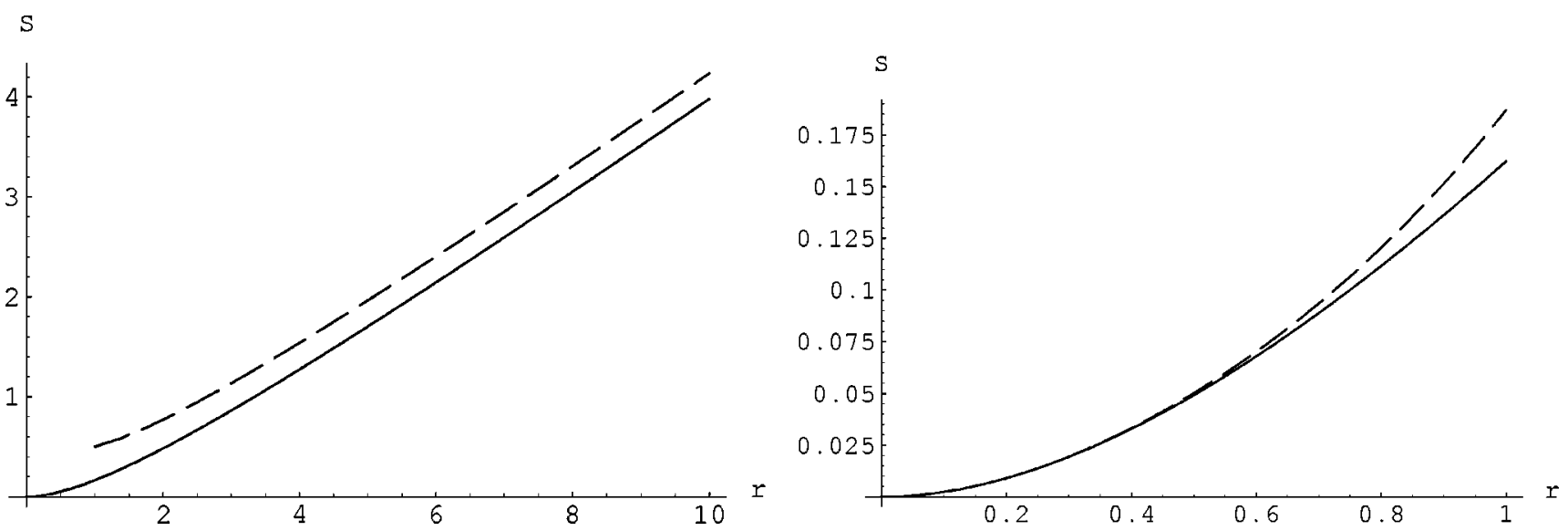

FIG. 1. $S(r)$ on the sphere compared to the first two terms of the asymptotic formula (6) and to the Taylor expansion (8).

this procedure yields the asymptotic expansion of $S(r)$ only up to a constant, because the condition $S(0)=0$ cannot be implemented. It begins as

$$
S(r) \approx \frac{r}{2}-\frac{\ln r}{3}-\frac{1}{15 r}-\frac{2}{105 r^{2}}-\frac{4}{315 r^{3}}+O\left(\frac{1}{r}\right)^{4}
$$

The presence of a subleading term $\ln r$ was to be expected, owing to the existence of a conformal anomaly for any Riemann surface, coming from the logarithmic divergence of the critical free energy. The logarithmic term of the free energy turns out to be

$$
W=-\frac{\chi}{12} \ln \left(\Lambda^{2} R^{2}\right)
$$

where $\Lambda$ is the UV cutoff, $R$ the size, and $\chi$ is the integral of the curvature divided by $4 \pi$, equal to the Euler-Poincaré number of the Riemann surface, according to the GaussBonnet theorem. The calculation of the conformal anomaly from the logarithmic divergence of the critical free energy is probably very old but was popularized by the development of string theory [5]. It was further discussed in Ref. [13], in the context of the Casimir energy. In the presence of mass, a term proportional to $\ln \left(\mathrm{m}^{2} / \mathrm{\Lambda}^{2}\right)$ must appear when the correlation length $m^{-1}$ becomes smaller than $R$. Therefore, it is not surprising to have the term $\ln r$ in the previous expansion. The concrete way in which it appears will be explained in Sec. IV, when we consider the heat-kernel derivation of the asymptotic expansion. This is a much more effective method to find the large $r$ behavior, capable of providing the generic form of the coefficients of the asymptotic series for arbitrary Riemann surfaces.

A plot of $S(r)$ is shown in Fig. 1, in comparison with the asymptotic behavior given by just the two growing terms in the previous formula (6). The agreement is quite remarkable, even almost down to $r=1$. The numerical value of the missing constant is approximately 0.254381 .

The small $r$ behavior of $S(r)$ is given by the power series expansion near $r=0$, which is easy to derive since $S^{\prime}(r)=$ $-r U^{\prime}(r)$. We obtain that

$$
\begin{aligned}
S(r)= & \frac{1}{4} r^{2}+\frac{1}{3}\left[2+\psi^{(2)}(1)\right] r^{3} \\
& +\frac{3}{16}\left[12+5 \psi^{(2)}(1)-\psi^{(2)}(2)\right] r^{4}+O(r)^{5},
\end{aligned}
$$

where $\psi^{(n)}(x)$ is the polygamma function. The behavior provided by the series truncated to this order is compared with the total $S(r)$ in the second plot of Fig. 1. The radius of convergence of the Taylor series is determined by the singular points of $U(r)$. Since the only singularities of $\psi(x)$ are simple poles for nonpositive integers, it is easy to see that the singularity closest to $r=0$ is a simple pole at $r=-2$, and hence the radius of convergence is 2 .

\section{THE RELATIVE ENTROPY OF THE TORUS}

We consider the torus as a rectangular box with periodic boundary conditions, which is the natural finite geometry in many applications. One can slightly generalize the boundary conditions by considering periodicity along two nonorthogonal directions, that is, by letting the box be a parallelogram. Although a parallelogram is in principle equivalent to a rectangle by an affine transformation, this is only true in real geometry, because in complex geometry such transformation is not allowed. Nevertheless, we shall consider a rectangle for simplicity and deduce the more general form from holomorphic factorization.

The partition function on the torus in the critical theory, $m=0$, is essentially the modulus of Dedekind's function $\eta(\tau)$. The classical proof of this result involves the use of the proper time representation and Poisson resummation, after analytical continuation in the manner of $\zeta$-function regularization, since the partition function is UV divergent. This method can be extended to the noncritical theory [16] (also see [17]). However, we favor a method similar to the one used for the sphere, where we calculate the energy $U(r)$. The substraction of the critical value will not be necessary since the UV divergence lends itself to straightforward identification.

Let $L$ and $M$ denote the periods in the horizontal and vertical directions, respectively. Then (see Appendix) 


$$
W(r)=\frac{M}{2} \sum_{l=-\infty}^{\infty} \epsilon(l)+\sum_{l=-\infty}^{\infty} \ln \left[1-e^{-M \epsilon(l)}\right]+C,
$$

where we have introduced the one-boson energies $\epsilon(l)$ $=\sqrt{(2 \pi l / L)^{2}+m^{2}}$ and where $C$ is a constant irrelevant for the relative entropy.

Now, we may notice that the previous expression for $W$ can be interpreted as the free energy of quantum $1 d$ bosons confined in a segment of length $L$ at finite temperature $T$ $=1 / M$ and constitutes a slight generalization of the expression for the cylinder considered before [2]. To prevent a divergence when $r=0$ we remove the zero mode $l=0$ and write

$$
W(r)=M \sum_{l=1}^{\infty} \epsilon(l)+2 \sum_{l=1}^{\infty} \ln \left[1-e^{-M \epsilon(l)}\right] .
$$

The UV divergences concentrate in the $1 d$ vacuum energy $E_{0}=\Sigma_{l=1}^{\infty} \epsilon(l)$. However, for $r=0$ they can be $\zeta$-regularized to give $E_{0}=(2 \pi / L) \zeta(-1)=-\pi /(6 L)$. The other term is the free energy of the bosonic excitations of the vacuum and is finite. For $r=0$ it combines with $E_{0}$ to yield $W \equiv-\ln Z$ $=\ln \eta(q)^{2}$, where $\eta(q)$ is the Dedekind function, $q$ $=\exp 2 \pi i \tau$, and the modular parameter is $\tau=i(M / L)$. In the case of a parallelogram the modular parameter is, of course, complex and $Z=1 /[\eta(q) \eta(\bar{q})]$.

Most of the discussion about the relative entropy of the cylinder in Ref. [2] holds as well for the torus. Hence, we change the notation for the vertical period, $M \rightarrow \beta$, in accord with the $1 d$ thermodynamic interpretation. The specific relative entropy is related to the quantum $1 d$ entropy of free bosons, $S_{q}$, as

$$
S(r ; L, \beta)=S(r ; L)-\frac{S_{q}}{2 L \beta}+\frac{\pi}{6 \beta^{2}},
$$

where

$$
\begin{gathered}
S(r ; L)=e_{0}(r ; L)-e_{0}(0 ; L)-r \frac{d e_{0}(r ; L)}{d r}, \\
e_{0}(r ; L):=\frac{E_{0}(r ; L)}{L} .
\end{gathered}
$$

The quantity $e_{0}(0 ; L)=-\pi /\left(6 L^{2}\right)$ is dual to $-\pi /\left(6 \beta^{2}\right)$. Note that

$$
S(r ; L)=\lim _{\beta \rightarrow \infty} S(r ; L, \beta)
$$

is the relative entropy of the cylinder. Of course, $\lim _{L \rightarrow \infty} S(r ; L)=\pi r / 2$. While $S(r ; L, \beta)$ is modular invariant, the $1 d$ quantum entropy $S_{q}$ comes only from the free energy of the bosonic excitations, namely, the second term in Eq. (10), and is not modular invariant. $S(r ; L, \beta)$ can be computed by the heat-kernel method, studied in the next section, in terms of a double series of Bessel functions. Its cylinder limit coincides with the single series of Bessel functions computed in Ref. [2]. On the other hand, it is feasible to obtain the perturbative expansion of $W$ near $m=0$ [16], and from it the expansion of $S(r ; L, \beta)$. The asymptotic expansion for large $m$ is just given by the value of $S$ for the plane and it comes from the first term, $S(r ; L)$, in Eq. (11), since the second one decays exponentially with $L$. A more precise description of this asymptotic behavior is provided by the heat-kernel method.

The quantum $1 d$ entropy of free bosons, $S_{q}$, can be related to the entanglement entropy of black holes in $1+1$ dimensions. The Euclidean version of Rindler space is just the punctured plane, which is conformally diffeomorphic to the cylinder. In addition, the entanglement entropy of this space can be computed with a path integral that reduces it to an ordinary entropy, when considered on the cylinder [7]. Therefore, one can use the formulas above, taking into account that the conformal transformation implies that the modular parameter becomes a function of the quotient of the two cutoffs for the radius, namely, $i / \tau=L / \beta=\ln \left(r_{1} / r_{0}\right)$. The complete expression of the cylinder entropy can be deduced from the results in Ref. [2]. The first term of the low mass expansion agrees with the one calculated by other methods in Ref. [7].

\section{HEAT-KERNEL TECHNIQUES}

The computation of thermodynamic quantities for the sphere or the torus, regardless of ulterior difficulties, begins with the preliminary step of determining the eigenvalues of the Laplacian. Unfortunately, even this preliminary step cannot be taken for an arbitrary compact Riemman surface. Therefore, one cannot start from a more or less formal but explicit expression of those quantities. Nevertheless, the situation is not hopeless for there is a powerful method to extract information on thermodynamic quantities, namely, the heatkernel representation of Green functions [3-6]. We now study this method, for the purpose of applying it to $g>1$ Riemman surfaces and for its own sake: actually, it provides useful insight for the sphere and the torus.

First, let us recall that

$$
W(m)=-\frac{1}{2} \operatorname{Tr}_{x, \vec{y}} \ln G(\vec{x}, \vec{y}), \quad U(m)=\frac{1}{2} \operatorname{Tr}_{x, y} G(\vec{x}, \vec{y})
$$

with $G(\vec{x}, \vec{y})$ the Green function of the Helmholtz equation, namely, $\left(\Delta+m^{2}\right) G(\vec{x}, \vec{y})=\delta(\vec{x}, \vec{y})$. If we introduce the Green function of the heat equation

$$
\Delta \phi=\frac{\partial \phi}{\partial t}
$$

called the heat kernel, $K(\vec{x}, \vec{y} ; t)$, we can express $G$ as a Laplace transform,

$$
G_{r}(\vec{x}, \vec{y})=\int_{0}^{\infty} d t e^{-r t} K(\vec{x}, \vec{y} ; t)
$$

after defining $r:=m^{2}$. Furthermore, 


$$
U(r)=\frac{1}{2} \int_{0}^{\infty} d t e^{-r t} \operatorname{Tr}_{x, \vec{y}} K(\vec{x}, \vec{y} ; t),
$$

and integrating over $r$,

$$
W(r)=-\frac{1}{2} \int_{0}^{\infty} \frac{d t}{t} e^{-r t} \operatorname{Tr}_{x, \vec{y}} K(\vec{x}, \vec{y} ; t)
$$

In the plane

$$
K(\vec{x}, \vec{y} ; t)=\frac{1}{4 \pi t} \exp -\frac{|\vec{x}-\vec{y}|^{2}}{4 t}
$$

so the specific energy is

$$
\frac{U(r)}{A}=\frac{1}{2} \int_{0}^{\infty} \frac{d t}{4 \pi t} e^{-r t},
$$

with $A=\int d^{2} x$ the total area. To avoid the logarithmic UV divergence at $t=0$ we may take a further derivative,

$$
\frac{U^{\prime}(r)}{A}=-\frac{1}{8 \pi} \int_{0}^{\infty} d t e^{-r t}=-\frac{1}{8 \pi r} .
$$

After integrating twice over $r$,

$$
\frac{W(r)}{A}=-\frac{1}{8 \pi}\left(r \ln r+C_{1} r+C_{2}\right),
$$

where $C_{1}$ and $C_{2}$ are two integration constants that are actually infinite due to UV divergences. Of course, this expression agrees with the one derived in [2] using UV regularization in momentum space.

In a general curved surface $\operatorname{Tr}_{x}, \vec{y} K(\vec{x}, \vec{y} ; t)$ $=\int d^{2} x K(\vec{x}, \vec{x} ; t)$ (the integrated heat kernel for coincident points). Note that $K(\vec{x}, \vec{x} ; t)$ explicitly depends on the point $\vec{x}$. However, for homogeneous surfaces it becomes independent of it. According to Eq. (16),

$$
\frac{U(r)}{A}=\frac{1}{2} \int_{0}^{\infty} d t e^{-r t} K(t),
$$

where $K(t)$ is the heat kernel for coincident points. If the eigenvalues of the Laplacian are available, $\Delta \phi_{n}=\gamma_{n} \phi_{n}$, the heat kernel is just

$$
K(t)=\sum_{n=0}^{\infty} e^{\gamma_{n} t}
$$

The first eigenvalue is $\gamma_{0} \equiv 0$, corresponding to the constant solution; the others are negative and ordered by their absolute value (in fact, $\gamma_{1}<-1 / 4$ [4]). Substituting for $K(t)$ in the integral representation (22) we recover the expression for the energy used before,

$$
\frac{U(r)}{A}=\frac{1}{2} \sum_{n=0}^{\infty} \frac{1}{-\gamma_{n}+r} .
$$

For example, we consider the torus, $\gamma_{n}=-(2 \pi)^{2}|k|^{2}$, where $k \in \Omega^{*}$, the lattice dual to $\Omega$, the one defining periodicity on the torus. Then we can use the Jacobi identity to write

$$
K(t)=\sum_{k \in \Omega^{*}} \exp \left[-(2 \pi)^{2}|k|^{2} t\right]=\frac{A}{4 \pi t} \sum_{\omega \in \Omega} \exp \left[-\frac{|\omega|^{2}}{4 t}\right] .
$$

The latter sum is $1+O[\exp (-1 / t)]$, reproducing the result for $U$ in the plane plus the corrections vanishing exponentially when $r \rightarrow \infty$.

Generically, the heat kernel $K(t)$ admits an asymptotic power series expansion at $t=0[18]$,

$$
K(t) \approx \frac{1}{4 \pi t}\left(1+\sum_{n=1}^{\infty} a_{n} t^{n}\right),
$$

in terms of some numerical coefficients determined by the Riemann curvature. This expansion, upon integration over $t$, leads to asymptotic expansions of thermodynamic quantities for large $r$. For the hyperbolic plane, it can be calculated from the integral of exp $-\nu^{2} t$ times the density of states of frequency $\nu[19,20]$ :

$$
K(t)=\frac{e^{-t / 4}}{2 \pi} \int_{0}^{\infty} d \nu \nu \tanh (\pi \nu) e^{-\nu^{2} t} .
$$

It yields

$$
K(t)=\frac{e^{-t / 4}}{4 \pi t}\left\{1-\sum_{n=1}^{\infty} \frac{1-2^{1-2 n}}{n !} B_{2 n} t^{n}\right\},
$$

where $B_{2 n}$ are the Bernoulli numbers. The expansion for the sphere is related to it by analytic continuation from the hyperbolic to the elliptic geometry; it has an additional $(-1)^{n}$ in the sum, and $e^{t / 4}$ instead of $e^{-t / 4}$.

From the expression (22) for the energy we can derive the heat-kernel integral for the specific relative entropy,

$$
\frac{S(r)}{A}=\frac{1}{2} \int_{0}^{\infty} \frac{d t}{t}\left[1-(1+r t) e^{-r t}\right] K(t),
$$

which can be easily shown to be convergent at $t=0$, taking into account the behavior of the heat kernel for small $t$ (26). For a compact surface, it also converges as $t \rightarrow \infty$ if we remove the zero mode, since then $K(t)$ decays exponentially, according to Eq. (23). To obtain the asymptotic $r \rightarrow \infty$ expansion we could be tempted to just substitute the aymptotic expansion (26) into this integral. However, we would find that the coefficients are given by divergent integrals as $t$ $\rightarrow \infty$. The problem is that we cannot prolong the aymptotic expansion (26) to $t=\infty$. However, the aymptotic expansion (26) can be substituted into the integral for $S^{\prime}(r)$,

$$
\frac{S^{\prime}(r)}{A}=-r \frac{U^{\prime}(r)}{A}=\frac{r}{2} \int_{0}^{\infty} d t e^{-r t} t K(t),
$$


to provide its asymptotic expansion $r \rightarrow \infty$, owing to Watson's lemma [21]. This expansion can in turn be subjected to indefinite integration to yield $S(r)$, of course, up to a constant:

$$
\frac{S(r)}{A} \approx-\frac{1}{8 \pi}\left(-r-a_{1} \ln r+\sum_{n=1}^{\infty} a_{n+1} \frac{(n+1) !}{n} r^{-n}\right) .
$$

We can obtain the whole asymptotic series of $S(r)$ for the sphere by just taking the coefficients from the corresponding asymptotic series of $K(t)$, given by Eq. (28) with $(-1)^{n}$ inserted. The coefficients of its succesive terms coincide with the ones in the previous expression (6) except for the one of the logarithmic term. This term, which was already remarked upon, is particularly interesting and we will now discuss it in more detail.

The logarithmic term in the asymptotic expansion of $S(r)$ and the conformal anomaly

The logarithmic term in the asymptotic expansion of $S(r)$ comes from the constant term in the asymptotic power series of $K(t)$ [see Eq. (26)], namely, $a_{1} /(4 \pi)$. In particular, formula (28) yields $a_{1}= \pm 1 / 3$ for the sphere and hyperbolic plane, respectively. If we substitute this constant for $K(t)$ in Eq. (30) we obtain

$$
\frac{S^{\prime}(r)}{A}=-r \frac{U^{\prime}(r)}{A}=\frac{r}{2} \int_{0}^{\infty} d t e^{-r t} t \frac{a_{1}}{4 \pi}=\frac{a_{1}}{8 \pi r} .
$$

(Notice the slight abuse of notation, for we deal here with truncated quantities.) For a curved compact homogeneous surface the Gauss-Bonnet theorem implies that $A=2 \pi|\chi|$ $=4 \pi|g-1|$, where $\chi$ is the Euler-Poincare number. Then the coefficient of the logarithmic term of the total value of $S(r)$ is

$$
\frac{a_{1} A}{8 \pi}=\frac{\chi}{12}=\frac{1-g}{6},
$$

only related to the topology of the Riemann surface. This result also holds for compact surfaces of variable curvature.

We can obtain as well the coefficient of the logarithmic term of $W$. Upon integrating $U^{\prime}(r)$ twice over $r$, we conclude that

$$
\frac{W(r)}{A}=\frac{a_{1}}{8 \pi} \ln r+C_{1} r+C_{2} .
$$

Hence we can try to connect with the critical value stated in Eq. (7) as follows. Let us return to the heat-kernel representation and perform the divergent integral for $W(r)$, from Eq. (17), with a lower cutoff. We find that

$$
\begin{aligned}
\frac{W(r)}{A} & =-\frac{a_{1}}{8 \pi} \int_{\epsilon}^{\infty} \frac{d t}{t} e^{-m^{2} t}=-\frac{a_{1}}{8 \pi} \Gamma\left(0, m^{2} \epsilon\right) \\
& =-\frac{a_{1}}{8 \pi}\left[-\ln \left(m^{2} \epsilon\right)-\gamma+O\left(m^{2} \epsilon\right)\right],
\end{aligned}
$$

where $\Gamma(0, x)$ is the incomplete gamma function. This agrees with the previous result. We can interpret the cutoff in $t$ as an UV cutoff, $\epsilon \sim \Lambda^{-2}$. By reinstating the radius of curvature we can then split $m^{2} \epsilon$ so that

$$
\ln \left(m^{2} \epsilon\right)=\ln r-\ln \left(\Lambda^{2} R^{2}\right) .
$$

The second term agrees with the critical value in Eq. (7), but the first term diverges as $r \rightarrow 0$. In fact, this limit is not meaningful since the logarithmic term arises in the asymptotic expansion for large $r$. Neither is it meaningful to utilize the asymptotic expansion of $K(t)$ [see Eq. (26)], to obtain the small- $r$ behavior; for $r=0$ the integrals for thermodynamic quantities are determined by the entire range of $t$ and not just by its asymptotic behavior, whether it is for small or large $t$. Nevertheless, Eq. (26) can still be utilized to determine the form of the UV divergence of $W(0)$. Indeed,

$$
W[0, \Lambda]=-\frac{A}{2} \int_{\Lambda^{-2}}^{\infty} \frac{d t}{t} K(t)
$$

implies that

$$
\Lambda \frac{\partial W[0, \Lambda]}{\partial \Lambda}=-A K\left(\Lambda^{-2}\right)=-\frac{A}{4 \pi}\left[\Lambda^{2}+a_{1}+O\left(\Lambda^{-2}\right)\right] .
$$

In the infinite-cutoff limit, $\Lambda R \gg 1$, we can discard the negative powers of $\Lambda$ and this equation is none other than the conformal anomaly equation for a rigid scale transformation (recall that $a_{1}$ is just the Riemann curvature divided by 3 ). Integration over $\Lambda$ yields the desired term,

$$
-\frac{A a_{1}}{4 \pi} \ln (\Lambda R)=-\frac{\chi}{6} \ln (\Lambda R)
$$

on a compact surface.

Notice however that $W(0)$ can also be calculated with zeta-function regularization and that it yields a finite result [6]. This may seem puzzling, for we have then lost track of the conformal anomaly. In any event, $W(0)$ is a pure number and its value is to some extent irrelevant. Nevertheless, in the massive case, zeta-function regularization also yields a finite $W(r)$, as we remark in the next section. But then the conformal anomaly can be extracted from the asymptotics of $W(r)$ for large $r$. Moreover, with any type of regularization, the asymptotics of $S(r)$ for large $r$ provides the conformal anomaly. This conclusion is not particularly interesting for the massive bosonic field theory, the only one considered here, but it may be very interesting for interacting theories.

We have concluded that the coefficient of the logarithmic term in the entropy must be the opposite of the conformal anomaly, that is, $\chi / 12$. While we had obtained $-1 / 3$ in Eq. (6) now we obtain $\chi / 12=1 / 6$. This discrepancy stems from having suppressed the zero mode in the calculation of $U(r)$ for the sphere. This contributes $1 / 2$ in any compact surface and $1 / 2-1 / 3=1 / 6$. We may make a little disgression here and recall that the calculation of the small- $t$ behavior of tensor Laplacians on compact Riemann surfaces provides a 
proof of the Riemann-Roch theorem [5,22]. In the case of the scalar Laplacian, $\Delta=\bar{\partial}^{\dagger} \bar{\partial}$, this theorem states that

$$
\mathcal{I}(\bar{\partial})=\operatorname{dim} \operatorname{Ker} \bar{\partial}-\operatorname{dim} \operatorname{Ker} \bar{\partial}^{\dagger}=1-g,
$$

where the adjoint $\bar{\partial}^{\dagger}=\nabla_{z}^{1}$ is the covariant derivative on forms. Then $\operatorname{Ker} \nabla_{z}^{1}$ is the space of Abelian differentials, with dimension $g$, and Ker $\bar{\partial}$ is the space of globally holomorphic functions (that is, constants), with dimension 1 (corresponding to the Laplacian zero mode). Their difference is $1-g=\chi / 2$. According to our discussion on the presence of logarithmic terms in $r$, we can interpret the zero mode as the logarithmic term for $r \rightarrow 0$ whereas the logarithmic terms found in the asymptotics $r \rightarrow \infty$ are related to the existence of nontrivial boundary conditions and hence to the existence of Abelian differentials. They subtract the right number $g$ from 1 such that the difference is proportional to the EulerPoincaré number. Indeed, we observe that, e.g., for the torus the zero-mode term $\ln \left[1-e^{-L m}\right] \approx \ln (m L)$ as $m \rightarrow 0$, while it vanishes exponentially in the opposite limit, $m \rightarrow \infty$. This is in accord with the torus being flat, so that $\chi=0$.

\section{THERMODYNAMIC QUANTITIES FOR COMPACT RIEMANN SURFACES OF HIGHER GENUS}

A compact Riemann surface of $g>1$ can be characterized by its fundamental group. When this surface is represented in its covering space, the hyperbolic plane, this surface gives rise to a tesselation of the hyperbolic plane, in which the tiles are identified by a discrete group of motions isomorphic to the fundamental group. Since the total group of motions of the hyperbolic plane is $S L(2, \mathrm{R})$, that group is one of its discrete noncommutative subgroups, which are called Fuchsian groups. This construction becomes analogous to the construction of the torus by factoring the plane by a lattice $\Omega$, where the fundamental group is $\mathbb{Z} \times \mathbb{Z}$. We have seen that there is an expression for the heat kernel of the torus as the kernel in the plane plus a series of powers of $\exp (-1 / t)[\mathrm{Eq}$. (25)]. There is a noncommutative analogue for $g>1$ : namely, the Selberg trace formula [4]

$$
\begin{aligned}
K(t)= & \frac{e^{-t / 4}}{2 \pi} \int_{0}^{\infty} d \nu \nu \tanh (\pi \nu) e^{-\nu^{2} t} \\
& +\frac{1}{2 A} \sum_{n=1}^{\infty} \sum_{\{\gamma\}} \frac{l_{\gamma}}{\sinh \left(n l_{\gamma} / 2\right)} \frac{e^{-t / 4}}{(4 \pi t)^{1 / 2}} \exp \left(-\frac{\left(n l_{\gamma}\right)^{2}}{4 t}\right) .
\end{aligned}
$$

In this formula $\{\gamma\}$ are the primitive conjugacy classes of the Fuchsian group and $l_{\gamma}$ is the length of the shortest geodesic along the corresponding noncontractible loop. The first term is just the integral representation of the heat kernel in the hyperbolic plane $H$ [see Eq. (27)]. It admits a different representation, more useful for computations, derived from the form of the corresponding Green function [4-6],

$$
K_{H}(t)=\frac{e^{-t / 4}}{(4 \pi t)^{3 / 2}} \int_{0}^{\infty} d b \frac{b e^{-b^{2} /(4 t)}}{\sinh (b / 2)} .
$$

Although the Selberg trace formula is a much more complicated formula than the Abelian one (25), it has the same structure; namely, it is a sum of the part corresponding to the infinite surface, now the hyperbolic plane, plus $O[\exp (-1 / t)]$ corrections due to the boundary conditions. As well as for the torus, these corrections give rise to exponentially vanishing terms which do not appear in the asymptotic expansion of the entropy around $r=\infty$. Therefore, all the higher genus Riemann surfaces share the same asymptotic expansion in $r$.

Formula (38) has been widely used to establish the modular dependence of partition functions in string theory $[6,22]$. The partition functions in string theory are those of critical theories, and Selberg's formula results in generalizations of the Dedekind function of the torus to $g>1$; they are called Selberg's zeta functions [4]. Although we are interested here in the massive case, the procedure to calculate the critical $W$ $[4,6]$ actually applies to the noncritical one as well. It consists of a part corresponding to the hyperbolic plane, which, is common for any homogeneous surface with $g>1$, and a part in terms of Selberg's zeta function, which accounts for the topology of the surface; namely,

$$
W(r)=-\frac{1}{2} \zeta_{r}^{\prime}(0)-(4 r+1)^{-1 / 4} \ln Z_{S}\left(\sqrt{r+\frac{1}{4}}+\frac{1}{2}\right) .
$$

The zeta function of the hyperbolic plane is

$$
\begin{aligned}
\zeta_{r}(x)= & \frac{A}{\Gamma(x)} \int_{0}^{\infty} \frac{d t}{t} t^{x} \frac{e^{-(r+1 / 4) t}}{(4 \pi t)^{3 / 2}} \int_{0}^{\infty} d b \frac{b e^{-b^{2} /(4 t)}}{\sinh (b / 2)} \\
= & \frac{A}{4 \pi^{3 / 2} \Gamma(x)}(1+4 r)^{3 / 4-x / 2} \\
& \times \int_{0}^{\infty} d b \frac{b^{x-1 / 2}}{\sinh (b / 2)} K_{3 / 2-x}\left(b \sqrt{r+\frac{1}{4}}\right) .
\end{aligned}
$$

Notice that it is a meromorphic function of $x$ with one single pole at $x=1$, as long as $|r|<1 / 4$. In the critical case the common part can be calculated exactly to yield

$$
\zeta_{0}^{\prime}(0)=2(g-1)\left[-\ln (2 \pi)+\frac{1}{2}-4 \zeta^{\prime}(-1)\right] .
$$

Selberg's zeta function is defined as

$$
Z_{S}(x)=\prod_{\gamma} \prod_{p=0}^{\infty}\left(1-e^{-(x+p) l} \gamma\right)
$$

The function $\zeta_{r}(x)$, as a regularization of $W$ on the hyperbolic plane, must lead to the asymptotic expansion of the relative entropy provided by Eqs. (28) and (29). The Selberg zeta function leads to the asymptotically vanishing corrections. 


\section{CONCLUSIONS}

We have seen that the general structure of the entropy of the free massive bosonic field theory on compact homogeneous Riemann surfaces consists of a part corresponding to the maximally symmetric surface, namely, to the sphere, the plane, or the hyperbolic plane, and a part due to the boundary conditions. The first part can be expressed as a complicated function, analytic in $r \in[0, \infty)$. Furthermore, it has an asymptotic expansion around $r=\infty$, which is fully computable. The second part embodies the topology and is more delicate to treat, but it vanishes exponentially as $r$ grows and therefore does not appear in the asymptotic expansion. The behavior of the entropy for small $r$ - the critical limit-is also calculable, in terms of a convergent series. It is strongly dependent on the global parameters defining the topological nature of the surface. Indeed, one can observe, for example, that the series for the sphere (8) has nothing to do with the one for the cylinder, obtained in Ref. [2]. The $k$ th term of the small- $r$ expansion is easily seen to be proportional to $\Sigma_{n}\left(-\gamma_{n}\right)^{-k}$, beginning with $k=2$. But for $g>1$ these sums cannot be computed analytically, because the Laplacian eigenvalues are not available.

The monotonic character of the entropy can be checked in our calculations. For large $m$ the entropy tends to $m^{2} /(8 \pi)$, independently of the topology of the surface, which is the value for the plane. However, the topology lets itself be felt in the subleading term, proportional to $\ln m$, which is actually related to the conformal anomaly. These two terms are the only ones divergent as $m \rightarrow \infty$. There is also a constant term in the asymptotic expansion, which cannot be determined exactly, however. The three terms together already provide a good approximation down to $m L \sim 1$. As we decrease $m$ we approach criticality and the entropy becomes very sensitive to large scale peculiarities of the surface, as already remarked, but one can check that it always remains monotonic.

We may wonder how much of the above can be generalized to higher dimensions or to interacting field theories. The spectrum of the Laplacian and the heat kernel for homogeneous spaces are well known [20]. In fact, the heat kernel is simpler in odd-dimensional homogeneous spaces than in even-dimensional ones, so the formulas for the threedimensional sphere or hyperbolic space turn out to be simpler as well. As regards massive interacting theories, an asymptotic expansion for large $m$ must exist in general and, moreover, the leading and subleading terms can also be studied. The leading term is always proportional to $\mathrm{m}^{2}$ for dimensional reasons, and its coefficient positive. To determine this coefficient one can use the thermodynamic-Bethe-ansatz computation of the universal bulk term of the free energy, as discussed before [2]. The subleading term is likely to be related to the conformal anomaly, $c(\chi / 6) \ln m$, where $c$ is the central charge of the conformal field theory for $m=0$. This may provide a new way to find the central charge of a model. The small- $m$ behavior can be studied with conformal perturbation theory, see Ref. [2]. However, it shall crucially depend on the nature of the surface, as well as on the particular field theory. This perturbation theory is presumably convergent for strongly relevant perturbations, like that of the free bosonic field theory.

\section{ACKNOWLEDGMENTS}

I acknowledge partial support under Grant PB96-0887. I thank Alvaro Domínguez for conversations and Matt Visser for a careful reading of a preliminary version of this manuscript.

\section{APPENDIX}

\section{Subtracted energy for the sphere}

We here perform the computation of the substracted energy for the sphere.

$$
U(r)=-\frac{r}{2}\left[\sum_{l=1}^{\infty} \frac{1}{[l(l+1)+r](l+1)}+\sum_{l=1}^{\infty} \frac{1}{[l(l+1)+r] l}\right]
$$

taking into account that

$$
\begin{gathered}
\sum_{l=1}^{\infty} \frac{1}{[l(l+1)+r] l}=\frac{1}{2 r}\left[2 \gamma+\left(1+\frac{1}{\sqrt{1-4 r}}\right) \psi\left(\frac{3-\sqrt{1-4 r}}{2}\right)+\left(1-\frac{1}{\sqrt{1-4 r}}\right) \psi\left(\frac{3+\sqrt{1-4 r}}{2}\right)\right], \\
\sum_{l=1}^{\infty} \frac{1}{[l(l+1)+r](l+1)}=\frac{-((1-\gamma)(1-4 r-\sqrt{1-4 r}))-(\sqrt{1-4 r}+2 r-1) \psi\left(\frac{1-\sqrt{1-4 r}}{2}\right)-2 r \psi\left(\frac{1+\sqrt{1-4 r}}{2}\right)}{(-1+\sqrt{1-4 r}) \sqrt{1-4 r} r},
\end{gathered}
$$

we have that 


$$
\begin{aligned}
U(r)= & \frac{1}{4}\left\{-2 \gamma-\left(1+\frac{1}{\sqrt{1-4 r}}\right) \psi\left(\frac{3-\sqrt{1-4 r}}{2}\right)-\left(1-\frac{1}{\sqrt{1-4 r}}\right) \psi\left(\frac{3+\sqrt{1-4 r}}{2}\right)\right. \\
& \left.+\frac{2\left((1-\gamma)(1-4 r-\sqrt{1-4 r})+(\sqrt{1-4 r}+2 r-1) \psi\left(\frac{1-\sqrt{1-4 r}}{2}\right)+2 r \psi\left(\frac{1+\sqrt{1-4 r}}{2}\right)\right)}{(-1+\sqrt{1-4 r}) \sqrt{1-4 r}}\right\} .
\end{aligned}
$$

The apparent pole at $r=0$ must cancel and a careful analysis shows that it does; the pole at $r=1 / 4$ cancels as well. Notice that when $r>1 / 4$ the argument of the digamma functions becomes complex. Nevertheless, $U(r)$ remains real and is an analytic function of $r$ at $r=1 / 4$.

\section{2. $W(r)$ for the torus}

We will let $L$ and $M$ denote the periods in the horizontal and vertical directions, respectively. Then

$$
W[m] \equiv-\ln Z[m]=\frac{1}{2} \sum_{l, n=-\infty}^{\infty} \ln \left[\left(\frac{2 \pi l}{L}\right)^{2}+\left(\frac{2 \pi n}{M}\right)^{2}+m^{2}\right],
$$

and

$$
U(r):=\frac{d W}{d r}=\frac{1}{2} \sum_{l, n=-\infty}^{\infty} \frac{1}{(l / L)^{2}+(n / M)^{2}+r},
$$

where $r:=(m / 2 \pi)^{2}$. To work out this sum we can use the known expansion of the hyperbolic cotangent in simple fractions [23],

$$
\operatorname{coth}(\pi x)=\frac{x}{\pi} \sum_{n=-\infty}^{\infty} \frac{1}{n^{2}+x^{2}}
$$

Hence,

$$
U(r)=\frac{1}{2} \sum_{l=-\infty}^{\infty} \frac{\pi M}{\sqrt{(l / L)^{2}+r}} \operatorname{coth}\left[\pi M \sqrt{(l / L)^{2}+r}\right] .
$$

Now we can obtain $W(r)$ by integration. Notice, however, that the series (A8) is divergent, so term by term integration is not warranted. However, the series for $U(r)-U(0)$ is convergent and one can apply term by term integration to that series. The ensuing series represents $W(r)-U(0) r$ minus a UV quadratically divergent constant. Of course, $U(0) r$ is the UV logarithmic divergence of $W(r)$. Since the UV divergences of $W(r)$ can be easily segregated, we can proceed with the integration term by term without further concern;

$$
\begin{aligned}
& \frac{M}{2} \int \frac{\pi d r}{\sqrt{(l / L)^{2}+r}} \operatorname{coth}\left[\pi M \sqrt{(l / L)^{2}+r}\right] \\
& \quad=\frac{M}{2} \int d \epsilon \operatorname{coth}\left(\frac{M}{2} \epsilon\right) \\
& \quad=\ln \sinh \left(\frac{M}{2} \epsilon\right),
\end{aligned}
$$

where we have introduced the one-boson energies $\epsilon(l)$ $=\sqrt{(2 \pi l / L)^{2}+m^{2}}$. Finally,

$$
\begin{aligned}
W(r)= & \sum_{l=-\infty}^{\infty} \ln \sinh \left(\frac{M}{2} \epsilon(l)\right)=\frac{M}{2} \sum_{l=-\infty}^{\infty} \epsilon(l) \\
& +\sum_{l=-\infty}^{\infty} \ln \left[1-e^{-M \epsilon(l)}\right]+C,
\end{aligned}
$$

where $C=-(\ln 2) \Sigma_{l=-\infty}^{\infty} 1$ is an irrelevant divergent constant. The other divergences appear in the first term and are of the form $C_{1}+C_{2} r$, as already remarked.
[1] J. Gaite and D. O'Connor, Phys. Rev. D 54, 5163 (1996).

[2] J. Gaite, Phys. Rev. Lett. 81, 3587 (1998); Phys. Rev. D 61, 045006 (2000).

[3] M. Kac, Am. Math. Monthly 73, 4, part II, 1 (1996); H. P. McKean and I. M. Singer, J. Diff. Geom. 1, 43 (1967); K. Stewartson and R. T. Waechert, Proc. Cambridge Philos. Soc. 69, 353 (1971).

[4] H. P. McKean, Commun. Pure Appl. Math. 25, 225 (1972).

[5] O. Alvarez, Nucl. Phys. B216, 125 (1983), and references therein.
[6] E. D’Hoker and D. H. Phong, Nucl. Phys. B269, 205 (1986); G. Gilbert, ibid. B277, 102 (1986); S. K. Blau and M. Clements, ibid. B284, 118 (1987).

[7] C. Callan and F. Wilczek, Phys. Lett. B 333, 55 (1994); C. Holzhey, F. Larsen, and F. Wilczek, Nucl. Phys. B424, 443 (1994); D. Kabat and M. J. Strassler, Phys. Lett. B 329, 46 (1994).

[8] J. L. Rosales, gr-qc/9712059.

[9] D. O'Connor and C. R. Stephens, Prog. Theor. Phys. 90, 747 (1993); B. L. Hu, in Proceedings of the Fourth International 
Workshop on Thermal Field Theory and Applications, Dalian, China, 1995, edited by Y. X. Gui and F. C. Khanna (World Scientific, Singapore, 1996), gr-qc/9606073; O. Iguchi, A. Hosoya, and T. Koike, Phys. Rev. D 57, 3340 (1998).

[10] D. Hochberg and J. Pérez-Mercader, Gen. Relativ. Gravit. 28, 1427 (1996); H. J. de Vega, N. Sánchez, and F. Combes, Astrophys. J. 500, 8 (1998).

[11] E. Alvarez and C. Gómez, Nucl. Phys. B541, 441 (1999); V. Balasubramanian and P. Kraus, Phys. Rev. Lett. 83, 3605 (1999).

[12] J. S. Dowker and R. Critchley, Phys. Rev. D 13, 3224 (1976); 15, 1484 (1977); S. Hawking, Commun. Math. Phys. 55, 133 (1977).

[13] S. Blau, M. Visser, and A. Wipf, Nucl. Phys. B310, 163 (1988).

[14] E. Elizalde, J. Math. Phys. 35, 3308 (1994); Ten Physical Applications of Spectral Zeta Functions (Springer-Verlag, Berlin,
1995).

[15] A. A. Bytsenko, G. Cognola, L. Vanzo, and S. Zerbini, Phys. Rep. 266, 1 (1996).

[16] H. Saleur and C. Itzykson, J. Stat. Phys. 48, 449 (1987); C. Itzykson and J. Drouffe, Statistical Field Theory (Cambridge University Press, Cambridge, England, 1989), Vol. II.

[17] C. Nash and D. O'Connor, Phys. Rev. Lett. 76, 1196 (1996).

[18] S. Minakshisundaram, J. Indian Math. Soc. 17, 158 (1953); P. Greiner, Arch. Ration. Mech. Anal. 41, 163 (1971).

[19] A. Ottewill and S. Takagi, Prog. Theor. Phys. 77, 310 (1987).

[20] R. Camporesi, Phys. Rep. 196, 1 (1990).

[21] C. M. Bender and S. A. Orszag, Advanced Mathematical Methods for Scientists and Engineers (McGraw-Hill, New York, 1978).

[22] L. Alvarez-Gaumé and P. Nelson, CERN-TH.4615/86 report.

[23] I. S. Gradshteyn and I. M. Ryzhik, Table of Integrals, Series, and Products (Academic, New York, 1980). 